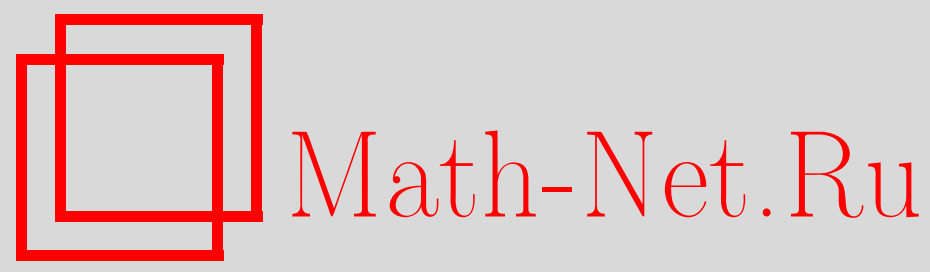

В. В. Грушин, О собственных значениях финитно возмущенного оператора Лапласа в бесконечных цилиндрических областях, Матем. заметки, 2004, том 75, выпуск $3,360-371$

DOI: https://doi.org/10.4213/mzm40

Использование Общероссийского математического портала Math-Net.Ru подразумевает, что вы прочитали и согласны с пользовательским соглашением http://www . mathnet.ru/rus/agreement

Параметры загрузки:

IP : 18.234 .156 .22

26 апреля 2023 г., 16:09:35 


\title{
О СОБСТВЕННЫХ ЗНАЧЕНИЯХ ФИНИТНО ВОЗМУЩЕННОГО ОПЕРАТОРА ЛАПЛАСА В БЕСКОНЕЧНЫХ ЦИЛИНДРИЧЕСКИХ ОБЛАСТЯХ
}

\section{В.В. Грушин}

\begin{abstract}
В работе приведены достаточные условия для существования собственных значений финитно возмущенного оператора Лапласа в бесконечной цилиндрической области и их асиптотика по малому параметру. Рассматриваются также аналогичные вопросы для трубок - деформированных цилиндров.

Библиография: 14 названий.
\end{abstract}

1. Введение. Пусть $M$ - ограниченная связная область с бесконечно гладкой $(\nu-$ 1)-мерной гранищей в $\mathbb{R}^{\nu}$ с координатами $y=\left(y_{1}, \ldots, y_{\nu}\right)$. В $\mathbb{R}^{\nu+1}$ с координатами $(x, y)=\left(x, y_{1}, \ldots, y_{\nu}\right)$ рассмотрим цилиндрическую область $\Omega=\{(x, y):-\infty<x<$ $+\infty, y \in M\}$. В $\Omega$ рассмотрим оператор

$$
H_{\varepsilon}=-\Delta_{D}^{\Omega}+\varepsilon A\left(x, y, \partial_{x}, \partial_{y}\right)+\varepsilon^{2} B\left(x, y, \varepsilon, \partial_{x}, \partial_{y}\right),
$$

где $\Delta_{D}^{\Omega}$ - оператор Лапласа в $\Omega, A$ и $B$ - линейные дифференциальные операторы второго порядка с бесконечно дифференцируемыми по совокупности переменных $(x, y) \in \bar{\Omega}$, $|\varepsilon|<\varepsilon_{0}$, и аналитическими по $\varepsilon$ коэффишиентами. Будем предполагать, что коэффициенты $A$ и $B$ финитны и их носители расположены в $[0, l] \times \bar{M}$ при некотором $l>0$. Обозначим через $\mathscr{H}_{D}^{2}(\Omega)$ подпространство функций из соболевского пространства $\mathscr{H}^{2}(\Omega)$, удовлетворяющих условию Дирихле $\left.u\right|_{\partial \Omega}=0$ (определение пространства $\mathscr{H}^{2}(\Omega)$ см., например, $\left[1\right.$, гл. 2]). Мы будем рассматривать $H_{\varepsilon}$ как непрерывньй оператор из $\mathscr{H}_{D}^{2}(\Omega)$ в $L_{2}(\Omega)$, либо как неограниченный оператор в $L_{2}(\Omega)$ с областью определения $\mathscr{H}_{D}^{2}(\Omega)$.

Основная задача этой статьи - получить достаточные условия для существования хотя бы одной собственной функции оператора $H_{\varepsilon}$ при достаточно малых $\varepsilon$ (т.е. слабо связанного состояния по терминологии из квантовой механики), а также получить асимптотические формулы для соответствующего собственного значения при $\varepsilon$ стремящемся к нулю (соответствуюшие результаты содержатся в теореме 1.1). Затем эти результаты применяются к вопросу о существовании собственных значений оператора Лапласа с условиями Дирихле в трубках - областях, представляюших собой искривленные бесконечные цилиндры (при $\nu=1$ цилиндрами называются полоски на плоскости). При переходе к подходящим образом выбранной системе координат трубка превращается в прямой цилиндр, а оператор Лапласа, записанный в таких координатах - в оператор вида (1.1), что позволяет для трубок использовать результаты, полученные для цилиндров. 
Изучению связанных состояний в трубках и приложениям этих вопросов к задачам квантовой механики посвящено большое количество работ, см. [2]-[7] и цитированную там литературу. В работах В. П. Маслова [8], [9], Маслова и Е. М. Воробьева [10] была построена асимптотика собственных функций и квазистационарных собственных значений уравнения $\Delta u+k^{2} u=0$ с краевыми условиями на эквидистантных кривых. Было показано, что подбирая нужным образом искривленность полоски и длину участка, где происходит искривление, можно добиться, чтобы существовало лишь одно собственное значение, т.е. резонатор имел лишь одну поперечную моду. Аналогичные вопросы исследовались для областей, ограниченных эквидистантными поверхностями. В работе В. В. Белова, С. Ю. Доброхотова, С. О. Синицьна [2] с помощью канонического оператора Маслова построены быстро осциллирующие решения уравнения Шрёдингера в тонких трубках; изучению связанных состояний в трубках посвящены работы П. Дюкло и П. Экснера [3], М. Энтина и Л. Магарила [4], Экснера [5]-[7].

Сформулируем основной результат статьи. Пусть $\left\{\chi_{n}\right\}-$ полная ортонормированная система собственных функций $-\Delta_{D}^{M}$ (т.е. оператора Лапласа со знаком "минус" в области $M$ с условием Дирихле),

$$
0<k_{0}^{2}<k_{1}^{2} \leqslant \cdots \leqslant k_{n}^{2} \leqslant k_{n+1}^{2} \leqslant \cdots
$$

- соответствующие собственные значения. Хорошо известно, что основное собственное значение $k_{0}^{2}$ невырожденно и $\chi_{0}(y)$ можно выбрать положительной в $M$ (см., например, [11]). Функции $\chi_{n}(y)$ можно выбрать вешественными. Мы будем использовать стандартное обозначение

$$
A_{m n}(x)=\int_{M} \bar{\chi}_{m}(y) A \chi_{n}(y) d y
$$

и аналогичным образом определяются $B_{m n}(x, \varepsilon)$. Зададим числа $\mu_{1}$ и $\mu_{2}$ формулами

$$
\begin{aligned}
\mu_{1}= & -\frac{1}{2} \int_{\mathbb{R}} A_{00}(x) d x \\
\mu_{2}= & -\frac{1}{2} \int_{\Omega} \bar{\chi}_{0}(y)\left(\mu_{1}[A, x]+A \int_{0}^{x}\left(x-x^{\prime}\right) A_{00}\left(x^{\prime}\right) d x^{\prime}+B_{00}(x, 0)\right) \chi_{0}(y) d x d y \\
& -\frac{1}{2} \sum_{n=1}^{\infty} \int_{\Omega} \bar{\chi}_{0}(y) A\left(\mathscr{E}_{\rho_{n}} * A_{n 0}\right) \chi_{n}(y) d y
\end{aligned}
$$

где $[A, x]$ - коммутатор оператора $A$ и оператора умножения на $x$, знак "звездочка" означает свертку функций,

$$
\mathscr{E}_{\rho_{n}}(x)=-\frac{1}{2 \rho_{n}} e^{-\rho_{n}|x|}, \quad \rho_{n}=\sqrt{k_{n}^{2}-k_{0}^{2}}
$$

Основным результатом статьи является следующее утверждение. 
Tеорема 1.1. Если $\operatorname{Re} \mu_{1}>0$ или $\operatorname{Re} \mu_{1}=0 u \operatorname{Re} \mu_{2}>0$, mo при некоторых $\varepsilon_{0}>0 u \mu_{0}>0$ для любого $0<\varepsilon<\varepsilon_{0}$ существует единственное собственное значение $\lambda$ оператора $H_{\varepsilon}$, удовлетворяющее неравенству

$$
\left|\lambda-k_{0}^{2}\right|<\mu_{0}^{2}
$$

Это собственное значение невырохденно и имеет при $\varepsilon \rightarrow 0$ асимптотику

$$
\lambda=k_{0}^{2}-\mu^{2}, \quad \mu=\varepsilon \mu_{1}+\varepsilon^{2} \mu_{2}+O\left(\varepsilon^{3}\right) .
$$

В случае вещественных коэффициентов операторов $A$ и $B$ такое собственное значение вещественно.

Если же $\operatorname{Re} \mu_{1}<0$ или $\operatorname{Re} \mu_{1}=0$ u $\operatorname{Re} \mu_{2}<0$, то при некоторых $\varepsilon_{0}>0 u \mu_{0}>0$ собственных значений, удовлетворяющих неравенству $(1.5), \partial \Omega_{\text {я }} 0<\varepsilon<\varepsilon_{0}$ не существует.

Доказательство этой теоремы будет дано в п. 4. В следующем пункте мы приведем примеры того, как эту теорему можно применять к оператору Лапласа (с условием Дирихле) в некоторых полосках и трубках.

2. Собственные значения оператора Лапласа в трубках. В этом пункте мы рассмотрим оператор Лапласа с условием Дирихле в трубках - областях, представляющих собой деформированные цилиндры. Собственные значения здесь могут возникать не за счет добавления возмущающих операторов к оператору Лапласа, а за счет изменения геометрии области. Однако и в таких случаях можно применять теорему 1.1 после перехода в систему координат, в которой область становится цилиндром, т.е. производя "вьпрямление" соответствующей трубки.

ПРИмЕР 2.1. Пусть $\Omega$ - область в $\mathbb{R}^{\nu+1}$, заданная неравенством

$$
|y|<d(1+\varepsilon b(x))
$$

где $b(x) \in C_{0}^{\infty}(\mathbb{R}), d>0$. Рассмотрим оператор Лапласа $\Delta_{D}^{\Omega}$ в $\Omega$ с областью определения $\mathscr{H}_{D}^{2}(\Omega)$. Пусть $k_{0}^{2}$ - наименьшее собственное значение оператора $-\Delta_{D}^{M}$, где $M-$ шар $|y|<d$ в $\mathbb{R}^{\nu}$. Положим

$$
\mu_{1}=k_{0}^{2} \int_{\mathbb{R}} b(x) d x
$$

ТЕОрема 2.1. Если $\mu_{1}>0$, то при некоторых $\varepsilon_{0}>0$ u $\mu_{0}>0$ для любого $0<\varepsilon<\varepsilon_{0}$ существует единственное собственное значение оператора $-\Delta_{D}^{\Omega}$, удовлетворяющее неравенству (1.5). Это собственное значение вещественно, невырожсденно и имеет при $\varepsilon \rightarrow 0$ асимптотику

$$
\lambda(\varepsilon)=k_{0}^{2}-\varepsilon^{2} \mu_{1}^{2}+O\left(\varepsilon^{3}\right) .
$$


ДокАЗАтЕльство. Так как оператор $\Delta_{D}^{\Omega}$ симметрический, он может иметь только вещественные собственные значения. После преобразования

$$
s=x, \quad \tau=y(1+\varepsilon b(x))^{-1}
$$

область $\Omega$ переходит в цилиндр $\widetilde{\Omega}=\{(s, \tau):|\tau| \leqslant d\}$, a $-\Delta_{D}^{\Omega}$ переходит в оператор

$$
\widetilde{H}_{\varepsilon}=-h^{-2} \partial_{\tau}^{2}-\left(\partial_{s}-\varepsilon \dot{b}(s) h^{-1} \partial_{\tau}\right)^{2}=-\partial_{s}^{2}-\partial_{\tau}^{2}+\varepsilon A+\varepsilon^{2} B,
$$

где $h=1+\varepsilon b(s)$, а $\dot{b}$ обозначает производную от $b(s)$ по $s$. Непосредственный подсчет показывает, что

$$
A=2 b \partial_{\tau}^{2}+2 \dot{b} \tau \partial_{s} \partial_{\tau}+\ddot{b} \tau \partial_{\tau}, \quad A_{00}=-2 k_{0}^{2} b(s)
$$

Формула (1.2) совпадает с (2.1) и утверждение теоремы 2.1 вытекает из теоремы 1.1.

Отметим, что $\Delta_{D}^{\Omega}$ совпадает с расширением по статочно малых $\varepsilon$ это вытекает из предложения 3.6, если его применить к оператору $h^{1 / 2} \widetilde{H}_{\varepsilon} h^{-1 / 2}$, которьй унитарно эквивалентен оператору $-\Delta_{D}^{\Omega}$.

Без предположения о малости $\varepsilon$ это можно доказать, используя соображения из замечания к предложению 3.5. Кроме того, результат теоремы 2.1 не изменится, если шар заменить произвольной областью $M$ в $\mathbb{R}^{\nu}$ с указанными во введении свойствами, а $\Omega$ определить как

$$
\Omega=\left\{(x, y): y(1+\varepsilon b(x))^{-1} \in M\right\} .
$$

ПримеР 2.2. Пусть на координатной плоскости $\left(x, y_{1}\right)$ в $\mathbb{R}^{\nu+1}$ имеется бесконечная несамопересекающаяся кривая $\Gamma$, заданная параметрическими уравнениями $x=X(s)$, $y_{1}=Y_{1}(s)$, причем $X(s)$ и $Y_{1}(s)$ принадлежат $C^{\infty}(\mathbb{R})$, и в качестве $s$ выбрана длина дуги (со знаком). Будем предполагать, что supp $\gamma \subset[0, l], l>0$, где $\gamma(s)=-\dot{X}(s) \ddot{Y}_{1}(s)+$ $\dot{Y}_{1}(s) \ddot{X}(s)$ - кривизна (со знаком) кривой Г. Последнее означает, что при $s \leqslant 0$ и $s \geqslant l$ кривая $\Gamma$ преврашается в прямые линии. Введем в окрестности $\Gamma$ "нормальные" ортогональные координаты, связанные с кривой $\Gamma$ :

$$
x=X(s)-\tau_{1} \dot{Y}(s), \quad y_{1}=Y_{1}(s)+\tau_{1} \dot{X}(s), \quad y_{j}=\tau_{j}, \quad 2 \leqslant j \leqslant \nu ;
$$

через $h$ обозначим якобиан $h=1+\tau_{1} \gamma(s)$. Рассмотрим в $\mathbb{R}^{\nu+1}$ искривленную трубку $\Omega$, которая получается как образ прямой трубки (цилиндра)

$$
\widetilde{\Omega}=\{(s, \tau):-\infty<s<+\infty, \tau \in M\},
$$

где $M$ удовлетворяет условиям из введения.

Будем предполагать, что отображение $(2.2)$ в $\overline{\widetilde{\Omega}}$ есть диффеоморфизм. Евклидова метрика записывается как

$$
d x^{2}+d y^{2}=\left(1+\gamma \tau_{1}\right)^{2} d s^{2}+d \tau^{2},
$$

поэтому оператор Лапласа в координатах $(s, \tau)$ имеет вид

$$
h^{-1} \partial_{s} h^{-1} \partial_{s}+h^{-1} \partial_{\tau} h \partial_{\tau}, \quad h=1+\gamma \tau_{1} .
$$

Мы будем рассматривать кривые $Г$ с малой кривизной, заменив $\gamma$ на $\varepsilon \gamma$. Тогда $h=1+$ $\varepsilon \gamma \tau_{1}$ и соответствующий оператор $\widetilde{H}_{\varepsilon}$ запишется в виде

$$
\widetilde{H}_{\varepsilon}=-\partial_{s}^{2}-\partial_{\tau}^{2}+\varepsilon A+\varepsilon^{2} B
$$


где

$$
\begin{aligned}
A & =2 \gamma \tau_{1} \partial_{s}^{2}+\dot{\gamma} \tau_{1} \partial_{s}-\gamma \partial_{\tau_{1}} \\
\left.B\right|_{\varepsilon=0} & =-3 \gamma^{2} \tau_{1}^{2} \partial_{s}^{2}-3 \dot{\gamma} \gamma \tau_{1}^{2} \partial_{s}+\gamma^{2} \tau_{1} \partial_{\tau_{1}}
\end{aligned}
$$

TЕорема 2.2. Если $\gamma \not \equiv 0$, то при некоторых $\varepsilon_{0}>0 u \mu_{0}>0$ для любого $0<\varepsilon<$ $\varepsilon_{0}$ существует единственное собственное значение $\lambda$ оператора $-\Delta_{D}^{\Omega}$ с условием Дирихле в трубке $\Omega$, построенной с помощью (2.2) и (2.3) по кривой Г с кривизной $\varepsilon \gamma, \gamma \in C_{0}^{\infty}(\mathbb{R})$, удовлетворяющее неравенству (1.5). Это собственное значение вещественно, невырохсдено и имеет при $\varepsilon \rightarrow 0$ асимптотику (1.6) с $\mu_{1}=0 u$

$$
\mu_{2}=-\frac{1}{8} \sum_{n=1}^{\infty} \rho_{n}^{4}\left(\tau_{1}\right)_{0 n}^{2} \int_{\mathbb{R}} \gamma\left(\mathscr{E}_{\rho_{n}} * \gamma\right) d s
$$

ДокАЗАТЕЛЬСТво. Подсчитаем $\mu_{1}$ и $\mu_{2}$ по формулам (1.2) и (1.3). Из (2.4) и (2.5) следует, что

$$
A_{n 0}(s)=-\gamma(s)\left(\partial_{\tau_{1}}\right)_{n 0}, \quad B_{00}(s, 0)=\gamma^{2}(s)\left(\tau_{1} \partial \tau_{1}\right)_{00}
$$

Интегрированием по частям получаются формулы

$$
\left(\partial_{\tau_{1}}\right)_{n 0}=-\left(\partial_{\tau_{1}}\right)_{0 n}, \quad\left(k_{n}^{2}-k_{0}^{2}\right)\left(\tau_{1}\right)_{0 n}=-2\left(\partial_{\tau_{1}}\right)_{n 0}, \quad\left(\tau_{1} \partial_{\tau_{1}}\right)_{00}=-\frac{1}{2},
$$

из которых, в частности, следует, что $A_{00}(s)=0$. Поэтому $\mu_{1}=0$ и

$$
\begin{aligned}
\mu_{2}= & \frac{1}{4} \int_{\mathbb{R}} \gamma^{2} d s \\
& +\frac{1}{2} \sum_{n=1}^{\infty} \int_{\mathbb{R}}\left(\partial_{\tau_{1}}\right)_{n 0}\left\{\left[2 \gamma\left(\mathscr{E}_{\rho_{n}} * \ddot{\gamma}\right)+\dot{\gamma}\left(\mathscr{E}_{\rho_{n}} * \dot{\gamma}\right)\right]\left(\tau_{1}\right)_{0 n}-\gamma\left(\mathscr{E}_{\rho_{n}} * \gamma\right)\left(\partial_{\tau_{1}}\right)_{0 n}\right\} d s d \tau .
\end{aligned}
$$

Интегрируя по частям и используя соотношение $\ddot{\mathscr{E}}_{\rho_{n}}-\rho_{n}^{2} \mathscr{E}_{\rho_{n}}=\delta(s)$, получаем

$$
\begin{aligned}
\mu_{2}= & \frac{1}{2}\left[\frac{1}{2}+\sum_{n=1}^{\infty}\left(\partial_{\tau_{1}}\right)_{n 0}\left(\tau_{1}\right)_{0 n}\right] \int_{\mathbb{R}} \gamma^{2} d s \\
& +\frac{1}{2} \sum_{n=1}^{\infty}\left(\partial_{\tau_{1}}\right)_{n 0}\left[\rho_{n}^{2}\left(\tau_{1}\right)_{0 n}-\left(\partial_{\tau_{1}}\right)_{n 0}\right] \int_{\mathbb{R}} \gamma\left(\mathscr{E}_{\rho_{n}} * \gamma\right) d s .
\end{aligned}
$$

В силу равенства Парсеваля для рядов Фурье и соотношений (2.7)

$$
\sum_{n=1}^{\infty}\left(\partial_{\tau_{1}}\right)_{n 0}\left(\tau_{1}\right)_{0 n}=\int_{M} \tau_{1} \chi_{0} \partial_{\tau_{1}} \chi_{0} d \tau=-\frac{1}{2}
$$

Используя (2.7), получаем формулу (2.6).

Если $\gamma \not \equiv 0$, то все интегралы в (2.5) отрицательны (это следует из равенства Парсеваля, если перейти к преобразованию Фурье), поэтому утверждение теоремы 2.2 следует из теоремы 1.1 (вещественность собственных значений также следует из того, что оператор Лапласа с условием Дирихле симметрический). Отметим, что формула, по существу эквивалентная (2.5), при несколько более общих предположениях на кривизну кривой была получена другим методом в [3], [6]. 
ПРИмЕР 2.3. Пусть $M$ - связная ограниченная область на плоскости $\left(y_{1}, y_{2}\right)$, граница которой состоит из конечного числа бесконечно гладких кривых. В пространстве $\mathbb{R}^{3}$ с координатами $\left(x, y_{1}, y_{2}\right)$ рассмотрим область $\Omega$, полученную поступательным перемещением $M$ параллельно оси $x$ с одновременным вращением вокруг оси $x$ на угол $\varepsilon \phi(x)$, где $\dot{\phi}(x) \in C_{0}^{\infty}(\mathbb{R})$. В подвижной системе координат $\left(s, \tau_{1}, \tau_{2}\right)$, связанной с исходной формулами

$$
s=x, \quad \tau_{1}=y_{1} \cos (\varepsilon \phi)+y_{2} \sin (\varepsilon \phi), \quad \tau_{2}=-y_{1} \sin (\varepsilon \phi)+y_{2} \cos (\varepsilon \phi),
$$

$\Omega$ превращается в цилиндр

$$
\widetilde{\Omega}=\{(s, \tau):-\infty<s<+\infty, \tau \in M\}
$$

В области $\Omega$, как и в предыдущих примерах, рассмотрим оператор $-\Delta_{D}^{\Omega}$, который при указанном преобразовании координат переходит в оператор

$$
\widetilde{H}_{\varepsilon}=-\partial_{\tau}^{2}-\left(\partial_{s}-\varepsilon \dot{\phi} L\right)^{2}, \quad L=\tau_{1} \partial_{\tau_{2}}-\tau_{2} \partial_{\tau_{1}} .
$$

Подсчитав $\mu_{1}$ и $\mu_{2}$ по формулам $(1.2),(1.3)$, где

$$
A=2 \dot{\phi} L \partial_{s}+\ddot{\phi} L, \quad B=-\dot{\phi}^{2} L^{2}
$$

получим, что $\mu_{1}=0$, а $\mu_{2}$ после аналогичных использованньг в примере 2.2 преобразований приводится к виду

$$
\mu_{2}=\frac{1}{2} \sum_{n=1}^{\infty}\left(L_{n 0}\right)^{2} \rho_{n}^{2} \int_{\mathbb{R}} \dot{\phi}\left(\mathscr{E}_{\rho_{n}} * \dot{\phi}\right) d s
$$

Если $\dot{\phi} \not \equiv 0$, то все интегралы в (2.8), как и в предыдущем примере, отрицательны, поэтому $\mu_{2} \leqslant 0$, причем $\mu_{2}=0$, если $L_{n 0}=0$ для всех $n$ и, следовательно, $L \chi_{0}=0$.

Так как $L=\partial_{\varphi}$, где $(r, \varphi)$ - полярные координаты на плоскости $\left(\tau_{1}, \tau_{2}\right)$, и $\chi_{0}$ положительна в $M$, то из этого вытекает, что любая окружность с центром в начале координат либо целиком содержится в $M$, либо с $M$ не имеет общих точек. Последнее возможно только в случае, когда гранища области состоит из одной или двух окружностей с центрами в начале координат, а это в свою очередь означает, что $\Omega$ представляет собой прямой (т.е. не искривленньй) цилиндр, а в прямом цилиндре собственных значений оператора $\Delta_{D}^{\Omega}$ нет.

Если $\Omega$ не является прямым цилиндром, то и из теоремы 1.1 мы получаем, что собственных значений, удовлетворяющих (1.5), у оператора $-\Delta_{D}^{\Omega}$ при $0<\varepsilon<\varepsilon_{0}$ нет ( $\varepsilon_{0}$ и $\mu_{0}$ определены в теореме 1.1).

3. Некоторые свойства возмущенного оператора Лапласа в бесконечном цилиндре. Пусть $\Omega$ - бесконечньй цилиндр в $\mathbb{R}^{\nu+1}$, удовлетворяющий условиям, сформулированньм во введении. В этом пункте мы изучим некоторые свойства оператора $H_{\varepsilon}$, определенного формулой (1.1). Предполагаются выполненными условия на операторы $A$ и $B$, сформулированные во введении. 
ПРЕДЛОЖЕНИЕ 3.1. Оператор $\Delta_{D}^{\Omega}$ осуществляет изоморфизм пространств $\mathscr{H}_{D}^{2}(\Omega) u L_{2}(\Omega)$.

Это утверждение по существу содержится в результатах работы В. А. Кондратьева [12]. Поэтому лиш коротко наметим схему этого доказательства.

1. Рассмотрим уравнение

$$
\Delta_{D}^{\Omega} u=f
$$

После преобразования $\Phi$ урье по переменной $x$ получаем

$$
\left(\Delta_{D}^{M}-\xi^{2}\right) \widetilde{u}(\xi, y)=\tilde{f}(\xi, y)
$$

и, следовательно,

$$
\widetilde{u}(\xi, y)=R(\xi) \widetilde{f}(\xi, y),
$$

где $R(\xi)$ - обратный оператор к $\Delta_{D}^{M}-\xi^{2}$.

2. Утверждение предложения теперь является следствием равенства Парсеваля и оценок для решений эллиптических краевых задач с параметром из работы М. С. Аграновича и М. И. Вишика [13].

ПРЕДЛОЖЕНИЕ 3.2. Для любого $u \in \mathscr{H}_{D}^{2}(\Omega)$ ряд Фурье

$$
u=\sum_{n=0}^{\infty} u_{n}(x) \chi_{n}(y), \quad u_{n}(x)=\int_{M} u_{n}(x, y) \bar{\chi}_{n}(y) d y
$$

сходится по норме в $\mathscr{H}_{D}^{2}(\Omega)$. Для любого $f \in L_{2}(\Omega)$ решение уравнения $\Delta_{D}^{\Omega} u=f$ дается формулами

$$
u=\sum_{n=0}^{\infty}\left(\mathscr{E}_{k_{n}} * f_{n}\right) \chi_{n}(y), \quad f_{n}(x)=\int_{M} f_{n}(x, y) \bar{\chi}_{n}(y) d y
$$

әде знак звездочки означает свертку функиий.

ДокАЗАТЕльСтво. Пусть $\Delta_{D}^{\Omega} u=f$; разложим $f$ в ряд $\Phi$ урье

$$
f=\sum_{n=0}^{\infty} f_{n}(x) \chi_{n}(x)
$$

Так как функции $u_{n}(x, y)=\left(\mathscr{E}_{k_{n}} * f_{n}\right) \chi_{n}(y)$ будут решениями уравнений $\Delta_{D}^{\Omega} u_{n}(x, y)=$ $f_{n}(x) \chi_{n}(y)$ и ряд (3.3) сходится в $L_{2}(\Omega)$, то в силу предложения 3.1 ряд (3.1) будет сходится к $u$, т.е. решение $u$ представляется в виде (3.1). В силу предложения 3.1 таким способом можно получить любое $f \in L_{2}(\Omega)$, что и доказьвает утверждение предложения 3.2 .

ПРЕДЛОЖЕНИЕ 3.3. Оператор $\Delta_{D}^{\Omega}$, как неограниченный оператор в $L_{2}(\Omega)$ с областью определения $\mathscr{H}_{D}^{2}(\Omega)$, самосопряжен.

Это утверждение вытекает из известной теоремы фон Неймана о том, что симметрический оператор в гильбертовом пространстве, область определения которого совпадает со всем пространством, самосопряжен. 
ПрЕДЛОЖЕНИЕ 3.4. При достаточно малых $\varepsilon$ оператор $H_{\varepsilon}$, определенный во введении, осуществляет изоморфизм пространств, указанных в предложсении 3.1 .

Это утверждение следует из того, что свойство операторов быть изоморфизмами сохраняется при малых по операторной норме возмущениях.

ПРЕДЛОЖЕНИЕ 3.5. Если оператор $H_{\varepsilon}$ симметрический на $C_{0}^{\infty}(\Omega)$, то при достаточно малых $\varepsilon$ он самосопряжен в $L_{2}(\Omega)$.

Действительно, если $H_{\varepsilon}$ симметрический на $C_{0}^{\infty}(\Omega)$ (такие дифференциальные операторы обычно называют формально самосопряженными), то оператор $H_{\varepsilon}$ будет симметрическим и на $\mathscr{H}_{D}^{2}(\Omega)$, что легко устанавливается интегрированием по частям. Теперь, учитьвая предложение 3.4 , опять можно воспользоваться теоремой фон Неймана.

Заметим, что утверждение о самосопряженности симметрического оператора $H_{\varepsilon}$ можно доказать и без предположения о малости $\varepsilon$, если наложить дополнительное условие эллиптичности задачи Дирихле для этого оператора. Это доказательство, использует локальные теоремы о гладкости решений эллиптических краевых задач и соответствующие оценки (см. $[1$, гл. 4 и 10]) в сочетании с подходящим разбиением единицы. Подробности мы опускаем, так как в данной работе мы ограничиваемся рассмотрением случая достаточно малых $\varepsilon$.

ПРЕДЛОЖЕНИЕ 3.6. Если оператор $H_{\varepsilon}$ симметрический, то при достаточно мальх в оператор $H_{\varepsilon}$ с областью определения $\mathscr{H}_{D}^{2}(\Omega)$ совпадает с расширением nо Фридрихсу оператора $H_{\varepsilon}$ на $C_{0}^{\infty}(\Omega)$.

Действительно, расширение по Фридрихсу представляет собой самосопряженный оператор (см., например, [14, гл. 5]). Из его построения ясно, что область определения этого расширения содержит $\mathscr{H}_{D}^{2}(\Omega)$, но на $\mathscr{H}_{D}^{2}(\Omega)$ в силу предложения 3.5 оператор $H_{\varepsilon}$ уже самосопряжен и его дальнейшее расширение с сохранением самосопряженности невозможно, что и доказьвает утверждение предложения 3.6.

4. Доказательство теоремы 1.1. Это доказательство представляет собой вариант обычной теории возмущений. Положим $\lambda=k_{0}^{2}-\mu^{2}$ и будем рассматривать уравнение

$$
H_{\varepsilon} u-k_{0}^{2} u+\mu^{2} u=0 .
$$

Пусть $\mathscr{L}_{0}$ - подпространство в $L_{2}(\Omega)$, состоящее из всех функций $\varphi(x) \chi_{0}(y), \varphi(x) \in$ $L_{2}(\mathbb{R}), \mathscr{L}_{0}^{\perp}$ - ортогональное дополнение к $\mathscr{L}_{0}, P_{0}$ - проектор на $\mathscr{L}_{0}$.

Лемма 4.1. При достаточно малых $\varepsilon$ и $\mu$ уравнение (4.1) имеет единственное решение $и(\varepsilon, \mu, x, y)$, удовлетворяющее следующим условиям:

а) $u(\varepsilon, \mu, x, y)$ можно представить в виде суммы

$$
u(\varepsilon, \mu, x, y)=u_{0}(\varepsilon, \mu, x) \chi_{0}(\eta)+U(\varepsilon, \mu, x, y)
$$

причем

$$
u_{0}(\varepsilon, \mu, x) \in \mathscr{H}_{\mathrm{loc}}^{2}(\mathbb{R}), \quad U(\varepsilon, \mu, x, y) \in \mathscr{H}_{D}^{2}(\Omega) \cap \mathscr{L}_{0}^{\perp}
$$

b) $u_{0}(\varepsilon, \mu, x)=e^{\mu x}$ npu $x \leqslant 0$. 
При этом $u_{0}(\varepsilon, \mu, x)$ и $U(\varepsilon, \mu, x, y)$ в указанных пространствах аналитически зависят от параметров $\varepsilon и \mu$.

Для доказательства рассмотрим болееширокую задачу: по заданным $f_{0}(x) \in L_{2}(0, l)$, $F(x, y) \in \mathscr{L}_{0}^{\perp}$ и числам $a_{0}, a_{1}$ найти такие

$$
v_{0}(\varepsilon, \mu, x) \in \mathscr{H}^{2}(0, l) \quad \text { и } \quad V(\varepsilon, \mu, x, y) \in \mathscr{H}_{D}^{2}(\Omega) \cap \mathscr{L}_{0}^{\perp}
$$

что

$$
\begin{gathered}
-\partial_{x}^{2} v_{0}+P_{0}\left(\varepsilon A+\varepsilon^{2} B\right)\left(v_{0} \chi_{0}+V\right)+\mu^{2} v_{0}=f_{0} \\
-\left(\Delta+k_{0}^{2}\right) V+\left(I-P_{0}\right)\left(\varepsilon A+\varepsilon^{2} B\right)\left(v_{0} \chi_{0}+V\right)+\mu^{2} V=F \\
v_{0}(0)=a_{0}, \quad \dot{v}_{0}(0)=a_{1} .
\end{gathered}
$$

Отображение

$$
\left(v_{0}, V\right) \rightarrow\left(f_{0}, F, a_{0}, a_{1}\right)
$$

непрерьвно в соответствующих пространствах. При $\varepsilon=0$ и $\mu=0$ эта задача однозначно разрешима, причем решение выражается явными формулами

$$
\begin{aligned}
v_{0}(0,0, x) & =a_{0}+a_{1} x-\int_{0}^{x}\left(x-x^{\prime}\right) f_{0}\left(x^{\prime}\right) d x^{\prime}, \quad 0 \leqslant x \leqslant l, \\
V(0,0, x, y) & =-\sum_{n=1}^{\infty}\left(\mathscr{E}_{\rho_{n}} * F_{n}\right) \chi_{n}(y)
\end{aligned}
$$

где $\mathscr{E}_{\rho_{n}}$ определены в (1.4). Таким образом, оператор (4.3) является изоморфизмом. Отсюда следует, что при достаточно малых $\varepsilon$ и $\mu$ изоморфизмом будет и отображение (4.3) причем обратньй оператор аналитически зависит от параметров $\varepsilon$ и $\mu$. Остается заметить, что решение $u(\varepsilon, \mu, x, y)$ получается из решения задачи $(4.2)$ при $f_{0}(x)=0$, $F(x, y)=0, a_{0}=1, a_{1}=\mu$, если продолжить $v_{0}(\varepsilon, \mu, x)$ на все $\mathbb{R}$ так, чтобы $v_{0}(\varepsilon, \mu, x) \in$ $\mathscr{H}_{\mathrm{loc}}^{2}(\mathbb{R})$ и $\ddot{v}_{0}-\mu^{2} v_{0}=0$ вне отрезка $[0, l]$, и положить

$$
u(\varepsilon, \mu, x, y)=v_{0}(\varepsilon, \mu, x) \chi_{0}(y)+V(\varepsilon, \mu, x, y)
$$

Лемма 4.2. Можсно найти такие $\varepsilon_{0}>0 u \mu_{0}>0$, что при $|\varepsilon|<\varepsilon_{0} u|\mu|<\mu_{0}$ уравнение (4.1) имеет единственное решение $u(\varepsilon, x, y)$, удовлетворяюшее условию а) и условиям

c) $u_{0}(\varepsilon, x)=u_{0}(\varepsilon, 0) e^{\mu x} n p u x<0 u u_{0}(\varepsilon, x)=u_{0}(\varepsilon, l) e^{-\mu(x-l)} n p u x>l$;

d) $u_{0}(\varepsilon, 0)=1$.

При этом существует аналитическая функиия $\mu(\varepsilon), \quad|\varepsilon|<\varepsilon_{0}$, такая, что $\mu(0)=0 u u(\varepsilon, x, y)=u(\varepsilon, \mu(\varepsilon), x, y)$. Ecли $A$ и $B$ имеют вещественные коэффиииенты, то $\mu(\varepsilon)$ при вещественных $\varepsilon$ будет вещ,ественной. 
ДоказАтельство. Решение $u(\varepsilon, x, y)$ получается из решения леммы 4.1 , если $\mu$ удовлетворяет уравнению

$$
G(\varepsilon, \mu)=0
$$

где

$$
G(\varepsilon, \mu)=\dot{u}_{0}(\varepsilon, \mu, l)+\mu u_{0}(\varepsilon, \mu, l) .
$$

В силу леммы 4.1 функция $G(\varepsilon, \mu)$ аналитична в окрестности точки $\varepsilon=0, \mu=0$. Легко видеть, что при $\varepsilon=0$ функция $u_{0}(0, x)$ совпадает с $e^{\mu x}$. Отсюда видно, что

$$
G(0,0)=0, \quad \partial_{\mu} G(0,0)=2 .
$$

Если $A$ и $B$ имеют вешественные коэффициенты, то при $\varepsilon$ и $\mu$ вещественных и $G(\varepsilon, \mu)$ будет вещественной. Теперь утверждение леммы следует из теоремы о неявной функции.

ЛЕмма 4.3. При $|\varepsilon|<\varepsilon_{0}$ число $\lambda$ из окрестности (1.5) точки $k_{0}^{2}$ тогда и только тогда является собственным значением оператора $H_{\varepsilon}$, когда $\operatorname{Re} \mu(\varepsilon)>0 u$ $\lambda=k_{0}^{2}-\mu^{2}(\varepsilon)$, әде $\varepsilon_{0}, \mu_{0} u \mu(\varepsilon)$ определены в лемме 4.2. При этом собственное значение $\lambda$ будет невырожденным, а соответствующую собственную функиию можсно взять в виде $u(\varepsilon, x, y)$, где $u(\varepsilon, x, y)$ определена в лемме 4.2 .

ДокАЗАТЕЛЬСтво. Собственные функции - это отличные от тождественного нуля решения уравнения $(4.1)$, принадлежашие $\mathscr{H}_{D}^{2}(\Omega)$, поэтому $u_{0}(x) \in \mathscr{H}^{2}(\mathbb{R})$ и удовлетворяет уравнению $\ddot{u}_{0}-\mu^{2} u_{0}=0$ при $x<0$ и $x>l$ (здесь $u$ - собственная функция). Из этого следует, что $u_{0}(x)$ удовлетворяет условию с) леммы 4.2 при $\operatorname{Re} \mu>0$.

Если $u_{0}(0)=0$, то отсюда следует, что $u_{0}(x)=0$ при $x<0$ и $\dot{u}_{0}(0)=0$. Представив $u$ в виде $u=u_{0}(x) \chi_{0}(y)+U(x, y)$, получим, что пара $\left(u_{0}, U\right)$ будет решением задачи (4.2) с $f_{0}=F=0, a_{0}=a_{1}=0$. В силу однозначной разрешимости задачи (4.2) из этого следует, что $U=0$ и $u_{0}(x)=0$ на $[0, l]$, а отсюда вытекает, что $u_{0}(x)=0$ и при $x>l$, т.е. $u \equiv 0$.

Так как собственная функция отлична от тождественного нуля, тем самым установлено, что $u_{0}(0) \neq 0$ и, следовательно, $u$ получается из решения $u(\varepsilon, x, y)$ леммы 4.2 умножением на константу. Тем самым лемма 4.3 доказана.

Собирая вместе результаты лемм 4.1-4.3, получаем следующее

ПРЕДЛОЖЕНИЕ 4.1. ДЛя некоторы $\varepsilon_{0}>0 u \mu_{0}>0$ существуют аналитическая при $|\varepsilon|<\varepsilon_{0}$ функиия $\mu(\varepsilon)$ и функиия

$$
u(\varepsilon, x, y)=u_{0}(\varepsilon, x) \chi_{0}(y)+U(\varepsilon, x, y)
$$

такие, что $u_{0}(\varepsilon, x)$ аналитически зависит от в в пространстве $\mathscr{H}^{2}(0, l)$ и удовлетворяет граничным условиям

$$
u_{0}(\varepsilon, 0)=1, \quad \dot{u}_{0}(\varepsilon, 0)=\mu(\varepsilon), \quad \dot{u}_{0}(\varepsilon, l)+\mu(\varepsilon) u_{0}(\varepsilon, l)=0 ;
$$

$U(\varepsilon, x, y)$ аналитически зависит от $\varepsilon$ в пространстве $\mathscr{H}_{D}^{2}(\Omega) \cap \mathscr{L}_{0}^{\perp}$. Собственное значение оператора $H_{\varepsilon}$ при $|\varepsilon|<\varepsilon_{0} u\left|\lambda-k_{0}^{2}\right|<\mu_{0}^{2}$ существует тогда и только тогда, когда $\operatorname{Re} \mu(\varepsilon)>0$. Eсли $\operatorname{Re} \mu(\varepsilon)>0$, то это собственное значение невырожденно, $\lambda=k_{0}^{2}-\mu^{2}(\varepsilon)$ и соответствующ,ю собственную функиию мохно взять в виде $u(\varepsilon, x, y)$.

Теперь для завершения доказательства теоремы 1.1 достаточно установить следующее 
ПРЕДЛОЖЕНИЕ 4.2. Функиия $\mu(\varepsilon)$, определенная в предложении 4.1, при $\varepsilon \rightarrow 0$ имеет разлохсение

$$
\mu(\varepsilon)=\varepsilon \mu_{1}+\varepsilon^{2} \mu_{2}+O\left(\varepsilon^{3}\right),
$$

где $\mu_{1}$ и $\mu_{2}$ вычисляются по формулам (1.2) и (1.3).

ДокАЗАТЕЛЬСтво. Учитьвая аналитическую зависимость функции $u(\varepsilon, x, y)$ из предложения 4.1, представим ее в виде

$$
u=u^{(0)}(x, y)+\varepsilon u^{(1)}(x, y)+\varepsilon^{2} u^{(2)}(x, y)+O\left(\varepsilon^{3}\right),
$$

подставим это разложение в уравнение (4.1) играничные условия (4.6), соберем коэффициенты при отдельных степенях параметра (что является стандартным подходом теории возмущений). Запишем полученные таким способом рекуррентные соотношения:

$$
\begin{aligned}
& \left(\Delta+k_{0}^{2}\right) u^{(0)}=0, \\
& \left(\Delta+k_{0}^{2}\right) u^{(1)}=A u^{(0)}, \\
& \left(\Delta+k_{0}^{2}\right) u^{(2)}=A u^{(1)}+B_{0} u^{(0)}+\mu_{1}^{2} u^{(0)}, \quad B_{0}=\left.B\right|_{\varepsilon=0}, \\
& u_{0}^{(0)}(0)=1, \quad \dot{u}_{0}^{(0)}(0)=0, \\
& u_{0}^{(1)}(0)=0, \quad \dot{u}_{0}^{(1)}(0)=\mu_{1}, \quad \dot{u}_{0}^{(1)}(l)+\mu_{1} u_{0}^{(0)}(l)=0, \\
& u_{0}^{(2)}(0)=0, \quad \dot{u}_{0}^{(2)}(0)=\mu_{2}, \quad \dot{u}_{0}^{(2)}(l)+\mu_{1} u_{0}^{(1)}(l)+\mu_{2} u_{0}^{(0)}(l)=0 .
\end{aligned}
$$

Уравнения (4.8)-(4.10) будем решать с помошью рядов Фурье по ортогональной системе $\left\{\chi_{n}\right\}$. Из уравнений нулевого приближения $(4.8),(4.11)$ находим, что $u^{(0)}(x, y)=\chi_{0}(y)$. Уравнения первого приближения $(4.9),(4.12)$ дают

$$
\begin{aligned}
& u_{0}^{(1)}(x)=\mu_{1} x+\int_{0}^{x}\left(x-x^{\prime}\right) A_{00}\left(x^{\prime}\right) d x^{\prime}, \quad 0 \leqslant x \leqslant l, \\
& u_{n}^{(1)}(x)=\left(\mathscr{E}_{\rho_{n}} * A_{n 0}\right)(x), \quad n \geqslant 1
\end{aligned}
$$

где $\mathscr{E}_{\rho_{n}}(x)$ определены в (1.4). Теперь из третьего уравнения в (4.12) получаем формулу (1.2). Наконец, из уравнений второго приближения (4.10), (4.13) следует, что

$$
u_{0}^{(2)}(x)=\mu_{2}+\int_{0}^{l}\left[\left(A u^{(1)}\right)_{0}+\left(B_{0} u^{(0)}\right)_{0}\right] d x+\mu_{1}^{2} l,
$$

где $(\cdot)_{0}$ означает коэффициент при $\chi_{0}$ в разложении по системе $\left\{\chi_{n}\right\}$. Поэтому третье условие в (4.13) приводит к соотношению

$$
2 \mu_{2}=-\int_{0}^{l}\left[\left(A u^{(1)}\right)_{0}+\left(B_{0} u^{(0)}\right)_{0}\right] d x+\mu_{1} \int_{0}^{l} x A_{00}(x) d x
$$

из которого и получается окончательная формула (1.3). Тем самым предложение 4.2 доказано.

В заключение автор благодарит профессора В. В. Белова и профессора С. Ю. Доброхотова, за обсуждение проблем, связанных со связанными состояниями в цилиндрических трубках и приложениями этих вопросов к задачам квантовой механики. 


\section{СПИСОК ЦИТИРОВАННОЙ ЛИТЕРАТУРЫ}

[1] Хермандер Л. Линейные дифференциальные операторы. М.: Мир, 1965.

[2] Белов В. В., Доброхотов С. Ю., Синицын С.О. Асимптотические решения уравнения Шрёдингера в тонких трубках // Тр. ин-та матем. и мех. УрО РАН. 2003. Т. 9. №1. C. 1-11.

[3] Duclos P., Exner P. Curvature-induced bound states in quantum waveguides in two and three dimensions // Rev. Math. Phys. 1995. V. 7. P. 73-102.

[4] Entin M. V., Magarill L. I. Electrons in twisted quantum wire // Phys. Rev. B. 2002. V. 66. P. 205308-1-205308-5.

[5] Exner P. Bound states in curved quantum waveguides // J. Math. Phys. 1989. V. 30. P. 2574-2580.

[6] Exner P. Bound states in quantum waveguides of a slowly decaying curvature // J. Math. Phys. 1993. V. 34. P. 23-28.

[7] Exner P. A quantum pipette // J. Math. Phys. A. 1995. V. 28. P. 5323-5330.

[8] Маслов В. П. Асимптотика собственных функций уравнения $\Delta u+k^{2} u=0$ с краевыми условиями на эквидистантных кривых и рассеяние электромагнитных волн в волноводе // Докл. AH CCCP. 1958. T. 123. № 4. C. 631-633.

[9] Maslov V. P. Mathematical aspects of integral optics // Russ. J. Math. Phys. 2001. V. 8. P. 83-180.

[10] Маслов В. П., Воробьев Е. М. Об одномодовых открытых резонаторах // Докл. АН CCCP. 1968. T. 179. № 3. C. 558-561.

[11] Рид М., Саймон Б. Методы современной математической физики. Т. 4. Анализ операторов. М.: Мир, 1982.

[12] Кондратьев В. А. Краевые задачи для эллиптических уравнений в областях с коническими и угловыми точками // Тр. ММО. 1967. Т. 16. С. 207-318.

[13] Агранович М. С., Вишик М. И. Эллиптические задачи с параметром и параболические задачи общего вида // УМН. 1964. Т. 19. № 3 (117). С. 53-161.

[14] Морен К. Методы гильбертова пространства. М.: Мир, 1965.

Московский государственный институт 